\title{
Self-Monitoring Blood Glucose in Type 2
}

\section{Diabetes: a Continuous Improvement Initiative}

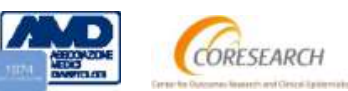

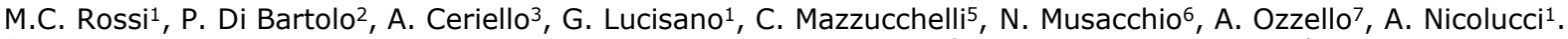

${ }^{1}$ Center for Outcomes Research and Clinical Epidemiology - CORESEARCH, Pescara, Italy; ${ }^{2}$ AUSL Romagna, Ravenna, Italy; ${ }^{3} I R C C S$ Multimedica, Sesto San Giovanni MI, Italy; ${ }^{5}$ IRCCS San Martino, Genova, Italy; ${ }^{6}$ AMD, Roma, Italy; ${ }^{7}$ Asl Torino 3, Pinerolo, Italy

\section{Background}

Self-monitoring blood glucose (SMBG) is a key tool for therapeutic decision and structured patient education. Despite there is evidence that SMBG improves metabolic control both in insulin-treated and in non insulin treated patients with type 2 diabetes (T2DM), the prescription and execution of SMBG is suboptimal and heterogeneous.

\section{Aim}

AMD Annals initiative is improving quality of diabetes care in Italy (Acta Diabetol 2015;52:557-71). This analysis aimed to assess the use of SMBG in patients with T2DM to evaluate frequency of SMBG, metabolic control, and hypo- and hyperglycemic episodes recorded in SMBG readings in the most frequent therapeutic schemes (with and without insulin).

\section{Method}

The standardized AMD Annals methodology has been applied for the extraction of the information contained in the electronic medical records (EMR). Information included clinical data, therapies and all SMBG values downloaded on EMR by different glucose meters routinely used by the patients. Sample was constituted by T2DM patients with at least one HbA1c value during the years 2014 and 2015 and with at least 1 available SMBG value measured in the 90 days before the HbA1c test. Frequency of SMBG, levels of fasting and post-prandial blood glucose (FBG and PPG), and values below 70 and 50 e over $300 \mathrm{mg} / \mathrm{dl}$ were defined as new quality of care indicators.

\section{Results}

Overall 21 centers and 13,331 patients (accounting for 35,657 HbA1c tests) were included in the analysis (figure 1). Indicators were assessed in the therapeutic schemes identified as the most prevalent (table 1 e figure 2):

Figure 1: Data flow-chart

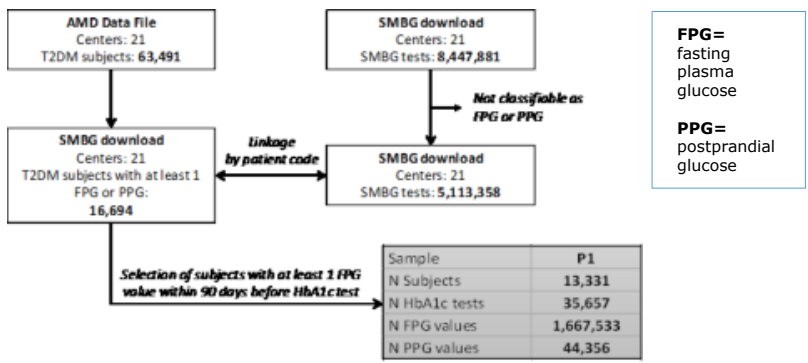

> $39.5 \%$ of SMBG tests could not be univocally classified as FBG or PPG (figure 1);

Of those values which could be univocally classified, over 95\% of SMBG tests were FBG values and less than 5\% were PPG values (figure 1).

- SMBG is frequently recommended also to patients not treated with insulin (table 1);

> Frequency of SMBG is suboptimal in all treatment schemes; even patients treated with schemes including insulin monitored their glucose less than two times per day (figure 2 - Panel A);

Pre-breakfast FBG values represented about $50 \%$ of all available FBG values in all treatment schemes (figure 2 - Panel A);

- The frequency of SMBG did not substantially differ among the most common schemes with oral agents, irrespective of the use of secretagogues (figure 2 - Panel A);

> The average FPG during three months was over $130 \mathrm{mg} / \mathrm{dl}$ in $49 \%$ to $88 \%$ of the cases in the different schemes;

Average PPG was over $140 \mathrm{mg} / \mathrm{dl}$ in $47 \%$ to $75 \%$ of the cases;

> Substantial proportions of cases have elevated FBG and PPG, even though average HbA1c levels were often acceptable (figure 2 - Panel B);

The use of therapeutic schemes including secretagogues was associated with a two to three-fold increased risk of glycemic values $<70 \mathrm{mg} / \mathrm{dl}$ as compared to OHA schemes without secretagogues (figure 2 - Panel C);

> The use of insulin markedly increased the risk of hypoglycemia, with a relevant difference between basal insulin+OHA and basal+short-acting insulins with or without OHA. The same trends were also found as for glycemic values $<50 \mathrm{mg} / \mathrm{dl}$ and $>300 \mathrm{mg} / \mathrm{dl}$.

Table 1: Most frequent therapeutic schemes and sample size

\begin{tabular}{|l|l|c|c|c|}
\hline Treatment group & Treatment scheme & $\begin{array}{c}\text { N } \\
\text { (HbA1c } \\
\text { test) }\end{array}$ & $\begin{array}{c}\text { Prevalence } \\
\text { within } \\
\text { scheme (\%) }\end{array}$ & $\begin{array}{c}\text { Code } \\
\text { in } \\
\text { Fig.2. }\end{array}$ \\
\hline \multirow{2}{*}{ Oral monotherapy } & Metformin only & 3188 & 63.8 & $\mathbf{1}$ \\
\hline \multirow{2}{*}{ Dual oral } & Sulphanulureas or Glinides only & 685 & 13.7 & $\mathbf{2}$ \\
\hline & Metformin + secretagogues & 2929 & 56.2 & $\mathbf{3}$ \\
\hline >=triple oral & Metformin + DPP-IV inhibitor & 612 & 11.9 & $\mathbf{4}$ \\
\hline \multirow{2}{*}{ GLP1-RAs + other } & Metformin + secretagogues + DPP-IV & 3132 & 72.5 & $\mathbf{5}$ \\
\hline \multirow{2}{*}{ Insulin+OHA } & Metformin + secretagogues + Acarbose & 337 & 7.8 & $\mathbf{6}$ \\
\hline & GLP1RA + Metformin + Secretagogue & 375 & 30.5 & $\mathbf{7}$ \\
\hline \multirow{2}{*}{ Insulin } & GLP1RA + Metformin & 516 & 42.0 & $\mathbf{8}$ \\
\hline & Basal insulin + Metformin + secretagogues & 3486 & 33.4 & $\mathbf{9}$ \\
\hline & Basal insulin + Short Acting insulin + Metformin & 1666 & 15.9 & $\mathbf{1 0}$ \\
\hline
\end{tabular}

Figure 2: Quality indicators of SMBG use and metabolic control by treatment scheme Number of SMBG tests per person-months by treatment scheme

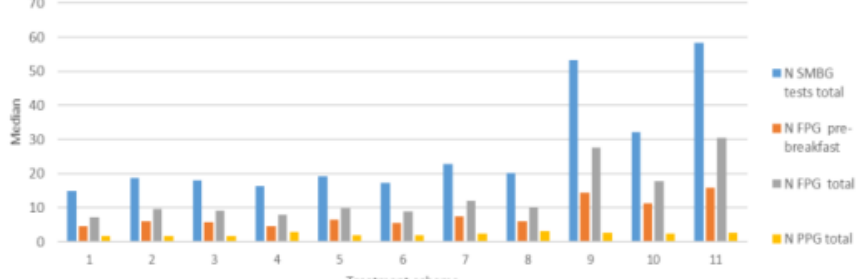

Mean levels of glycemia from SMBG tests
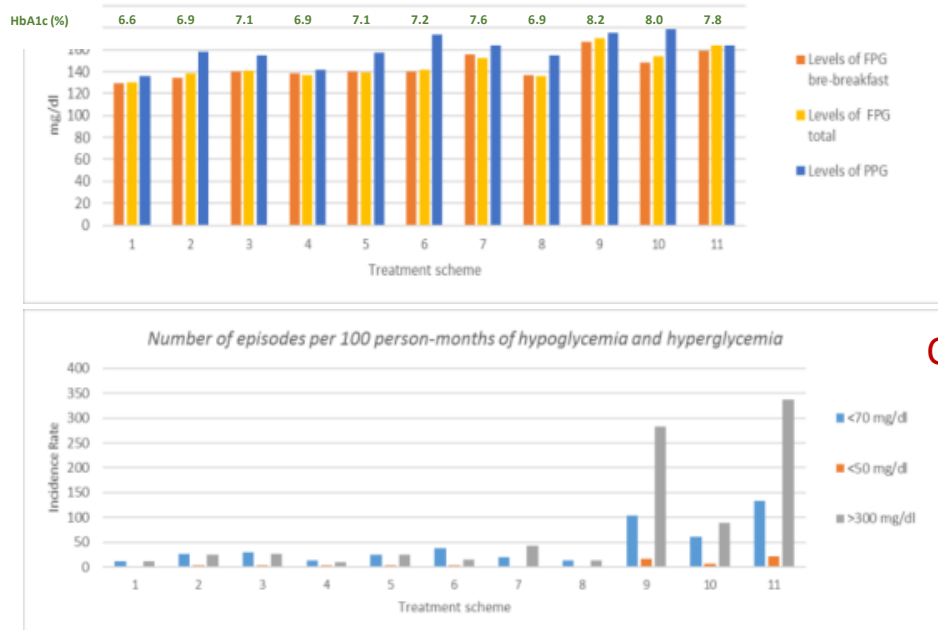

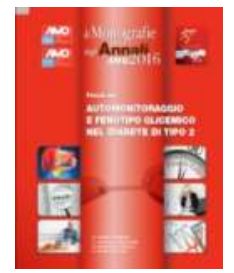

Free dowload

http://aemmedi.it/wpcontent/uploads/2016/09 /Monografia 2016 Auto monitoraggio.pdf [Italian]

\section{Conclusions}

$>$ There is an urgent need to improve SMBG use in type 2 diabetes;

$>$ AMD Annals initiative is working to increase the culture and the appropriate use of SMBG.

Investigators: Nuzzi Annamaria - Alba, Canale Investigators: Muzzi Annamaria - Alba, Canale (CN), Velussi Mario - Aurisina (TS), Spanu Francesca - Cagliari, Del Buono Andrea - Cellole (CE), Santantonio Graziano - Civitavecchia (RM), Magro Giampaolo - Cuneo, Garrapa Gabriella - Fano $(\mathrm{PU})$, Antenucci Daniela - Lanciano (CH), Laudato Mario - Maddaloni (CE), Saitta Giovanni - Messina, Stroppiana Mauro - Nizza Monferrato (AT), D'Agati Francesco - Palermo, Anichini Roberto - Pescia (PT), Di Bartolo Paolo - Ravenna, Rastelli Emilio Riccione (RN), Leotta Sergio - Roma, Felace Giuseppe - Spilimbergo (PN), Lalli Carlo - Spoleto $(P G)$, Tonutti Laura - Udine 